Draft VERSion OCTOBER 22, 2018

Preprint typeset using $\mathrm{LAT}_{\mathrm{E}} \mathrm{X}$ style emulateapj v. 08/22/09

\title{
HALO ASSEMBLY BIAS IN HIERARCHICAL STRUCTURE FORMATION
}

\author{
Neal Dalal ${ }^{1}$, Martin White ${ }^{2}$, J. Richard Bond ${ }^{1}$ and Alexander Shirokov ${ }^{1}$ \\ Draft version October 22, 2018
}

\begin{abstract}
We investigate the origin of halo assembly bias, the dependence of halo clustering on assembly history. We relate halo assembly to peak properties measured in the Lagrangian space of the initial linear Gaussian random density field, and show how these same Lagrangian properties determine large-scale bias. We focus on the two regimes where assembly bias has been observed to be significant: at masses very large and very small compared to the nonlinear mass scale. At high masses, we show that assembly bias is expected from the statistics of the peaks of Gaussian random fluctuations, and we show that the extent of assembly bias found in N-body simulations of rare halos is in excellent agreement with our theoretical prediction. At low masses, we argue that assembly bias largely arises from a sub-population of low mass halos whose mass accretion has ceased. Due to their arrested development, these halos naturally become unbiased, in contrast to their anti-biased peers. We show that a simple toy model incorporating these effects can roughly reproduce the bias trends found in N-body simulations.

Subject headings: cosmology:theory - methods:numerical - dark matter: merging histories - galaxies: clusters
\end{abstract}

\section{INTRODUCTION}

Hierarchical structure formation is a basic prediction of the highly successful cold dark matter cosmological model, and a key element of hierarchical growth is the formation of bound virialized objects called dark matter halos. Dark matter halos are not distributed uniformly throughout the universe, but rather compose a beaded filamentary network, sometimes referred to as the cosmic web (Bond et al. 1996). The clustering of the rare, massive dark matter halos is enhanced relative to the general mass distribution (Kaiser 1984; Efstathiou et al. 1988; Cole \& Kaiser 1989; Bond et al. 1991; Mo \& White 1996; Sheth \& Tormen 1999), an effect known as bias. The more massive the halo, the larger the bias. As a result, the mass of halos hosting a given population of objects is sometimes inferred by measuring their degree of clustering - allowing a statistical route to the notoriously difficult problem of measuring masses of cosmological objects. There are many applications for statistical mass determination, including (for example) cluster mass-observable self-calibration (e.g. Lima \& Hu 2004; Wu et al. 2008) or reconstruction of the halo occupation distribution of galaxies or quasars (e.g. Cooray \& Sheth 2002).

Since halos of a given mass can differ in their formation history, properties and large-scale environment ${ }^{3}$, a natural question arises: do these details affect halo clustering? Numerical simulations are now able to produce samples with sufficient statistics to test for the dependence of clustering on history, properties and environment and recent numerical simulations reveal subtle dependences of halo clustering on formation redshift, in-

\footnotetext{
${ }^{1}$ Canadian Institute for Theoretical Astrophysics, University of Toronto, 60 St. George St., Toronto, ON, M5S 3H8, Canada

2 Department of Astronomy, University of California, Berkeley, CA 94720

3 The large-scale environment of a halo refers to the density, smoothed on some suitably large scale, e.g. $8 h^{-1} \mathrm{Mpc}$.
}

ternal structure and spin (Gao, Springel \& White 2005; Wechsler et al.|2006; Harker et al.|2006; Bett et al.|2007; Wetzel et al.|2007; Jing. Suto \& Mo 2007; Gao \& White 2007; Angulo, Baugh \& Lacey 2008)

Using a marked correlation function, Sheth \& Tormen (2004) found that close halo pairs tend to have earlier formation times than more distant pairs, work which was extended and confirmed by Harker et al. (2006). Gao. Springel \& White (2005) found that later forming, low-mass halos are less clustered than typical halos of the same mass at the present. Wechsler et al. (2006) and Wetzel et al. (2007) found a similar dependence upon halo formation time (defined in a slightly different way), showing that the trend reversed for more massive halos and that the clustering depended on halo concentration. Recently, Gao \& White (2007) showed that a wide variety of halo properties correlate with bias, including spin and substructure content, while Li et al. (2008) showed that different definitions of halo age, when applied to the same halo samples, give different amounts of assembly bias.

What remains unclear from this numerical work is the physical origin of this effect. Gao, Springel \& White (2005) argued that assembly bias is inconsistent with the Press \& Schechter (1974) model, which explicitly predicts that halo abundance (and hence clustering) depends only upon halo mass and no other internal properties related to assembly history. Attempts to explain assembly bias based on the Press-Schechter approach (i.e. the excursion set using a sharp $k$-space filter) would therefore appear doomed from the outset, however several workers have explored modifications to the PressSchechter model in the hopes of accounting for assembly bias. Sandvik et al. (2007) considered excursion set mass functions using barrier heights determined from the ellipsoidal collapse model of Bond \& Mvers (1996), but were unable to account for the magnitude of assembly bias seen in simulations. Mo et al. (2005) suggested that "pre-heating", arising from the partial collapse of struc- 
ture into sheets and filaments, could suppress mass accretion onto halos within those sheets and filaments. This mechanism would clearly be incapable of explaining assembly bias in high-mass halos, which collapse before the formation of sheets and filaments (Bond et al. 1996), and Sandvik et al. (2007) found that this mechanism fails in low-mass halos as well. Desjacques (2007) investigated the effects of environment on the Bond \& Myers (1996) ellipsoidal collapse model, but could not reproduce the sign reversal of assembly bias between high and low masses, or the magnitude of assembly bias seen in N-body simulations.

In this paper, we will argue that many aspects of assembly bias may be understood quite simply. In particular, we show that the statistics of Gaussian random fields require significant assembly bias in high-mass halos, and we show using N-body simulations that the clustering of massive halos matches precisely the assembly bias that we predict. At low halo masses, we argue that assembly bias arises largely due to the cessation of mass accretion onto a sub-population of small halos. These non-accreting halos then naturally become unbiased over time, and therefore cluster more strongly than the majority of halos of similar mass, which are anti-biased.

\section{BIAS}

In this section, we briefly review previous results on biasing and assembly bias.

We start by assuming that massive dark matter halos arise from the collapse of peaks in the initial (linear) density field; this allows us to infer many of the properties of halo statistics using the statistics of gaussian fields (Press \& Schechter 1974; Bardeen et al. 1986; Bond et al. 1991; Bond \& Myers 1996). High peaks of the density field will in general cluster more strongly than the average matter clustering, which leads to halo bias. In the linear bias model, we directly relate the halo fluctuations to the local matter density fluctuations,

$$
\delta_{h}=b \delta
$$

where $\delta \equiv \delta \rho / \bar{\rho}$ is the overdensity and $b$ is the bias parameter. On scales over which this expression is valid ${ }^{4}$, we may express halo two-point functions in terms of the matter two-point function, scaled by appropriate powers of the bias. For example, the halo power spectrum would become $P_{h}(k)=b^{2} P_{m}(k)$.

The clustering of halos reflects the clustering of initial peaks only in part, however. Another contribution to halo clustering comes from the motion of peaks along large-scale matter flows. At late times, the halo overdensity becomes

$$
\delta_{h}(a)=\delta_{\mathrm{L}}+\delta_{m}(a)
$$

where the Lagrangian overdensity $\delta_{\mathrm{L}}$ corresponds to the clustering of the peaks in the initial density field, and the second term $\delta_{m}$ reflects the motion of the peaks due to advection along matter flows. On sufficiently large scales, in which we can treat the motion of the peaks as tracing the average bulk motion, the second term is

\footnotetext{
4 This approximation should be valid on scales where the matter correlation is small, $\xi(r) \ll 1$ or $\Delta^{2}(k)=k^{3} P(k) / 2 \pi^{2} \ll 1$ (Kaiser 1984). In general the scale-dependence of the bias will extend to larger scales the more biased the halos.
}

simply the matter overdensity. Then the (Eulerian) bias becomes

$$
\begin{aligned}
b(a) & =\delta_{h}(a) / \delta_{m}(a)=1+\delta_{\mathrm{L}} / \delta_{m}(a) \\
& =1+b_{\mathrm{L}}(a) .
\end{aligned}
$$

Note that we explicitly include the time dependence of the matter overdensity, but that the Lagrangian overdensity of peaks is not time dependent: it simply reflects the clustering of peaks in the initial conditions. Therefore, over time, the clustering associated with motion will dominate over the clustering from the initial conditions. That is, if we can treat the halos as test particles, we expect their Lagrangian bias to decay with time inversely with the linear growth factor, $b_{\mathrm{L}}(a) \propto 1 / D(a)$ (e.g. Fry 1996; Tegmark \& Peebles 1998).

Henceforth, we will focus on the Lagrangian bias $b_{\mathrm{L}}$, noting that the conversion to Eulerian bias $b$ is simply given by Eqn. (3) on linear scales. The next step is to determine $b_{\mathrm{L}}$. From Eqn. (1), we can think of bias as the rate at which the number of halos or peaks changes as the background density is varied. If we associate dark matter halos with rare peaks above some high threshold density, then it becomes easy to see why massive halos are biased. In the Press-Schechter model, for example, the (Lagrangian) number density of halos is

$$
n_{\mathrm{PS}} \propto \frac{\delta_{c}}{\sigma^{2}} \exp \left(-\frac{\delta_{c}^{2}}{2 \sigma^{2}}\right),
$$

and changing the background density level by $\delta$ is equivalent to changing the threshold density to $\delta_{c}-\delta$. Then the bias becomes

$$
b_{\mathrm{PS}}=n^{-1} \frac{d n}{d \delta}=-n^{-1} \frac{d n}{d \delta_{c}}=\frac{\delta_{c}}{\sigma^{2}}-\frac{1}{\delta_{c}}
$$

(Cole \& Kaiser 1989). This precise expression applies only for the Press-Schechter mass function, but its qualitative features are generally valid. At high masses $\left(\sigma \ll \delta_{c}\right)$, peaks above threshold are much more clustered than matter, because changing the background density level dramatically changes the probability out on the tail of the Gaussian distribution. In contrast, at low masses $\left(\sigma \gg \delta_{c}\right)$, halos are anti-biased $\left(b_{\mathrm{L}}<0\right)$ because raising the background density level reduces the number of peaks at low mass, by converting them to higher mass.

Clearly, many aspects of the mass dependence of bias may be inferred by consideration of the initial density peaks from which halos form. We can largely understand the clustering of Eulerian halos found in complex nonlinear simulations by adopting a Lagrangian viewpoint, and examining the properties of peaks of the initial linear density field. As mentioned in the introduction, simulations have shown that bias depends upon additional halo properties besides mass, such as assembly history or structural parameters like concentration. The philosophy of this paper will be to assume that this additional dependence may also be understood from the statistics of the initial Gaussian peaks. Accordingly, we will study Nbody halos found in simulations described below, using a Lagrangian viewpoint.

\section{SIMULATION AND POST-PROCESSING}

We have performed N-body simulations of structure formation in scale-free cosmologies, with $\Omega_{m}=1$ and 
power-law power spectra. We have chosen to run scalefree models rather than more realistic $\Lambda \mathrm{CDM}$ models in order to simplify the problem as much as possible, and remove possible effects from late-time cosmic acceleration or a varying spectral index. On scales far from the box size and far from the Nyquist frequency, the only relevant scale in the problem is $M_{\star}(a)$, the characteristic nonlinear mass scale satisfying $\sigma\left(M_{\star}, a\right)=\delta_{c}=1.68$. If the power spectrum slope is $n$, such that $P(k) \propto k^{n}$, then the rms mass fluctuations scale like $\sigma(M) \propto M^{-(3+n) / 6}$. Because the linear growth factor behaves as $D(a)=a$ for this critical-density cosmology, the nonlinear mass scales like $M_{\star}(a) \propto a^{6 /(3+n)}$. Since the nonlinear mass varies rapidly with expansion factor $a$, by examining halo properties at multiple redshifts we can study halo properties over a wide range of $M / M_{\star}$ (Efstathiou et al. 1988; Wechsler et al. 2006).

In this paper, we will focus on the largest simulation we ran, with $1024^{3}$ particles, using a power spectral index $n=-2$. We normalized the power spectrum such that $\sigma(M, a)=10 a M^{-1 / 6}$, where the mass $M$ is measured in number of particles. The simulation was evolved using the adaptive $\mathrm{P}^{3} \mathrm{M}$ code gracos (Shirokov \& Bertschinger 2005), using a Plummer softening length $\varepsilon_{P}=0.1$ times the mean interparticle spacing. The simulation was started at $a=0.005$, and 100 outputs were generated, spaced evenly in $\log a$ from $a=0.0825$ to $a=1$, containing phase-space coordinates (comoving positions and peculiar velocities) and ID's for each particle.

The phase space data for each output is used to find the (sub-)halos in a two-step process. First we generate a catalog of halos using the Friends-of-Friends (FoF) algorithm (Davis et al. 1985) with a linking length of $b=0.2$ times the mean inter-particle spacing. This procedure partitions the particles into equivalence classes by linking together all particles separated by less than a distance $b$, with a density of roughly $\rho>3 /\left(2 \pi b^{3}\right) \simeq 100$ times the background density. We keep all halos with more than 32 particles.

We identify subhalos of the FOF groups using a new implementation of the Subfind method of Springel et al. (2001) which defines subhalos as gravitationally selfbound aggregations of particles bounded by a density saddle point. After some experimentation with different techniques we found this method gave a good match to what would be selected "by eye" as subhalos. We use a spline kernel with 16 neighbors to estimate the density and keep all subhalos with more than 20 particles. We call the most massive subhalo in any FoF group the central halo. The other subhalos we refer to as satellites.

For each subhalo we compute and store a number of properties including the bound mass, velocity dispersion, peak circular velocity $\left(v_{c}\right)$, total potential energy, position and velocity. Since mass loss can be quite extreme for some subhalos we will use $v_{c}$ rather than mass to quote subhalo 'size'. The mass, peak circular velocity and potential are highly correlated, however since our subhalos are not spherical, there is a non-trivial scatter between $v_{c}$ and mass at fixed time.

A merger tree is computed from the set of subhalo catalogs by identifying for each subhalo a "child" at a later time. We process 4 consecutive simulation outputs at a time and for each subhalo in the earliest output we define its child as the subhalo at a later time which maximizes

$$
\alpha=\ln ^{-1}\left(\frac{a_{2}}{a_{1}}\right)\left[1-\frac{\left|M_{1}-M_{2}\right|}{M_{1}+M_{2}}\right] \sum_{i \in 2} \phi_{1 i}^{2}
$$

where $a_{1}$ and $M_{1}$ are the scale-factor and mass of the progenitor, $a_{2}$ and $M_{2}$ are the candidate halo at the later time, $\phi_{1 i}$ is the potential of particle $i$ computed using the particles in subhalo 1 and the sum is over all particles in the progenitor that also lie in the candidate. The weighting by $\phi^{2}$ ensures that the "core" particles in the progenitor are given more weight than the less bound particles. We found that weighting by $\phi^{2}$ gave better results than weighting by $\phi$, but higher powers of $\phi$ did not perform appreciably better than $\phi^{2}$. We find $\sim 95 \%$ of the subhalos have a child in the next time step, with one or two percent skipping one or more output times. A similar method for subhalo tracking has been employed by Springel et al. (2005), Faltenbacher et al. (2005), Kravtsov et al. (2004), Allgood et al. (2006), and Harker et al. (2006).

In addition to the Eulerian halo properties mentioned above, we also require Lagrangian properties of halos, such as their centroids or mean initial velocities. From the initial conditions for the simulation, we have the initial density field $\delta(\boldsymbol{x})$, the initial comoving displacement field $\boldsymbol{d}(\boldsymbol{x})$ satisfying $\delta+\nabla \cdot \boldsymbol{d}=0$, the peculiar velocity field, which in the Zeldovich approximation for $\Omega_{m}=1$ is $\boldsymbol{v}=a H \boldsymbol{d}$, and the strain field $\mathbf{S}=\nabla \boldsymbol{d}$. Note that $\operatorname{Tr} \mathbf{S}=-\delta$. These fields are all computed on the same grid as the initial (undisplaced) particle positions, by Fourier transform of the initial density field. For example, the strain field $S_{i j}$ is computed by FFT of $\delta_{\boldsymbol{k}} k_{i} k_{j} / k^{2}$. We would like to average these quantities over the Lagrangian volumes occupied by the halos. We may do so using the unique ID's for each particle. For each field $F$, we compute the average $\langle F\rangle$ over every halo by a simple average over the halo's particles:

$$
\langle F\rangle_{i}=N_{i}^{-1} \sum_{j=1}^{N_{i}} F\left(\boldsymbol{x}_{i, j}\right)
$$

where $\boldsymbol{x}_{i, j}$ is the Lagrangian position of the $j^{\text {th }}$ particle of halo $i$. Lastly, we will also require derivatives of these smoothed properties with respect to mass, e.g. $d\langle\delta\rangle / d(\log M)$. We use the merger tree described above to compute derivatives with respect to mass. For every halo, we move along the most massive branch of its merger tree for a factor of 2 in mass, tabulating mass $M$ and the average $\langle F\rangle$. We then fit a linear expansion, $F=F_{0}+F^{\prime} \Delta(\log M)$ in order to measure the derivative $F^{\prime} \equiv d F / d \log M$.

Lastly, we will determine bias parameters in Fourier space. We measure the matter power spectrum $P(k)$, the halo auto-power spectrum $P_{h}(k)$, and the halomatter cross spectrum $P_{c}(k)$, and define the halo bias as $b=P_{c}(k) / P(k)$. We use the ratio of the cross-spectrum rather than the halo auto-spectrum, because the former is less sensitive to shot noise in the halo counts.

\section{HIGH MASS HALOS: $M \gg M_{\star}$}

We first focus on halos well above the nonlinear mass scale, $M \gg M_{\star}$, where $\sigma(M) \ll \delta_{c}$. These halos are par- 


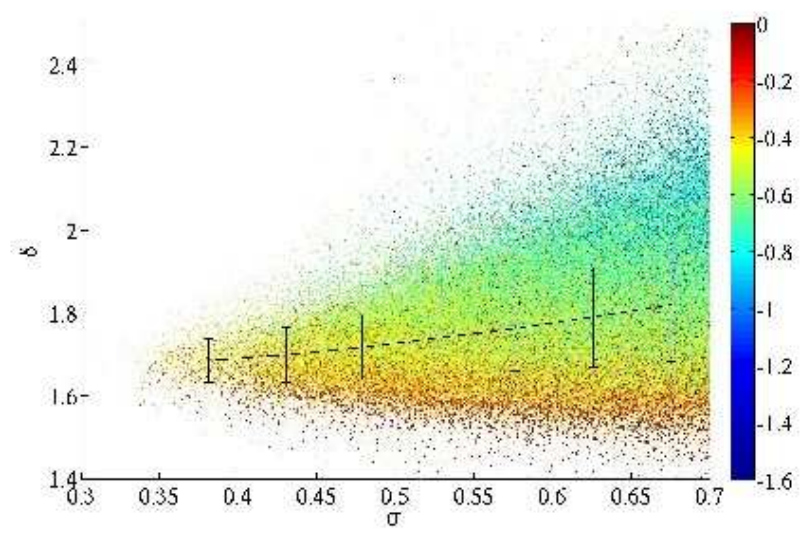

FIG. 1.- Average Lagrangian overdensity $\langle\delta\rangle$ for halos as a function of $\sigma(M)$. In the high mass limit, $\sigma \rightarrow 0$, collapse becomes spherical and $\langle\delta\rangle \rightarrow \delta_{c}=1.68$. Point colors correspond to peak curvature, $d\langle\delta\rangle / d \log M$, as denoted in the colorbar on the right. The solid black curve and error bars depict the mean and standard deviation of $\delta$ in bins of $\sigma$.

ticularly simple : because they are so massive, they dominate their environments, and because they are far out on the tail of the probability distribution of $\delta$, the mass dependence of their bias scales like $b \simeq \delta_{c} / \sigma^{2}$. Anti-biasing from larger-scale halos should be negligible. Another key simplification in this regime is that the collapse of these rare peaks is nearly spherical (Bardeen et al. 1986, Appendix $\mathrm{C}$ ). Because the collapse is roughly spherical, we expect the collapse threshold predicted by the spherical collapse model (Gunn \& Gott 1972) to be a good approximation: $\delta_{c} \approx 1.68$. This expectation is borne out by our $\mathrm{N}$-body results. In figure 1, we plot the average linearly evolved overdensity for FOF halos well above $M_{\star}$, and indeed we find $\langle\delta\rangle \simeq 1.7$ for the $\gtrsim 3 \sigma$ peaks. Note that multiple different redshifts and halo masses are plotted in this figure.

The validity of $\delta_{c} \approx 1.68$ for halos in the regime $\sigma \ll 1$ allows us easily to estimate the halo bias as $b \sim \delta_{c} / \sigma^{2}$ as mentioned above. This is the average over all peaks of a given mass, which marginalizes over any other peak parameters which could affect bias. One obvious additional parameter which the bias must depend upon is the curvature of the peak, which we will parametrize by $s=d\langle\delta\rangle / d(\log M)$. To see this, note that we can estimate the large-scale background density $\delta_{b}$ in which the halos reside by a Taylor expansion: $\delta_{b} \approx \delta+\Delta(\log M) \times d \delta / d(\log M)+\ldots$ Two peaks of the same mass $M$ and peak height $\delta$ but different peak curvatures $s_{1}>s_{2}$ (i.e. $\left|s_{1}\right|<\left|s_{2}\right|$ ) will tend to live in different environments: peak 1 will tend to have a larger background density than peak 2 , and therefore will have a larger bias. It is straightforward to estimate the dependence of bias on curvature. Writing $\nu=\delta / \sigma, x=s / \sigma_{s}$, and the cross-correlation coefficient $\gamma=\langle\nu x\rangle$, the bias becomes $(\text { Bardeen et al. 1986 })^{5}$

$$
b_{\mathrm{L}} \approx \sigma^{-1} \frac{\nu-\gamma x}{1-\gamma^{2}}
$$

We can easily sketch a derivation of this expression following the argument of Kaiser (1984). Let us com-

5 Bardeen et al. (1986) parametrized curvature not with $d \delta / d \log M$ as we do, but rather with the closely related $\nabla^{2} \delta$.

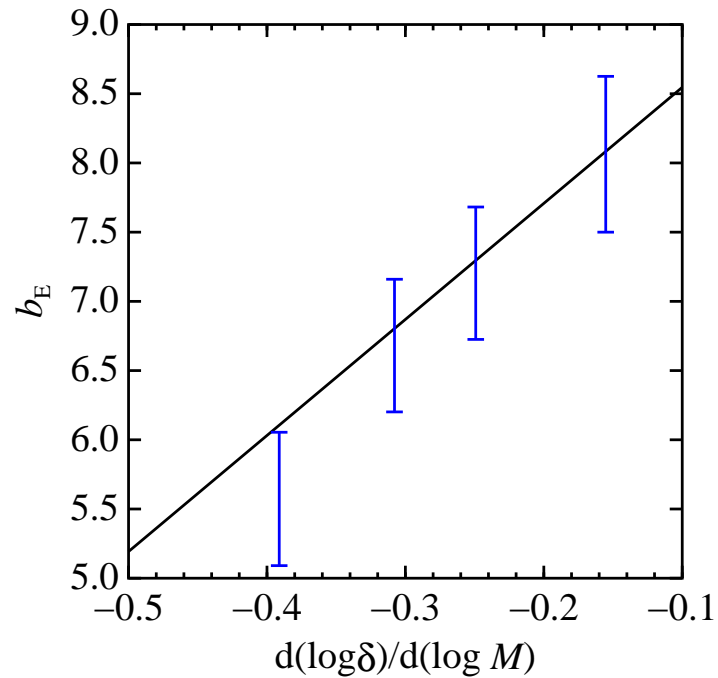

FIG. 2.- Eulerian bias $b_{\mathrm{E}}=1+b_{\mathrm{L}}$ for halos with 110-130 particles at $a=0.1089$, as a function of peak curvature. These halos have $\sigma=0.49$, and are roughly $\sim 1600 M_{\star}$ at this redshift. Halos are binned into quartiles of $d \log \delta / d \log M$, and halo bias in each bin is measured by the ratio of the halo-matter cross-spectrum to the matter power spectrum. The solid black curve shows Eqn. (8).

pute the correlation function for regions above a high threshold $\nu_{t}$. Consider regions 1 and 2, each of size $R$ and separated by distance $r_{12}$, with peak heights $\nu_{1}, \nu_{2}$ and curvatures $x_{1}, x_{2}$. These Gaussian fields are described by a covariance matrix with diagonal elements $\left\langle\nu^{2}\right\rangle=\left\langle x^{2}\right\rangle=1$. Of the off-diagonal elements, only $\left\langle\nu_{1} \nu_{2}\right\rangle=\psi\left(r_{12}\right)$ and $\left\langle\nu_{1} x_{1}\right\rangle=\left\langle\nu_{2} x_{2}\right\rangle=\gamma$ are important; long-range correlations involving derivatives of the density field fall off more quickly than these terms by powers of $R / r_{12}$. We compute the two-point correlation of regions with $\nu>\nu_{t}$ by integrating the Gaussian probability over $\nu_{1}>\nu_{t}, \nu_{2}>\nu_{t}$. If we are uninterested in the curvature $x$, then we first marginalize over $x_{1}$ and $x_{2}$, leaving a $2 \times 2$ covariance matrix with off-diagonal element $\psi$, and we find that the two-point function for regions above threshold becomes $\xi_{\mathrm{pk}}=\nu_{t}^{2} \psi$, giving a Lagrangian bias $b_{\mathrm{L}}=\nu_{t} / \sigma$ (Kaiser 1984). We can similarly compute the two-point function for regions above threshold with a specified curvature $x$ by repeating this calculation, but not marginalizing over $x_{1}$ and $x_{2}$. Recalling that the one-point probability distribution for $\nu$ at a given $x$ is a Gaussian centered at $\langle\nu \mid x\rangle=\gamma x$ with variance $1-\gamma^{2}$, we change variables from $\nu$ to $\nu^{\prime}=(\nu-\gamma x) /\left(1-\gamma^{2}\right)^{1 / 2}$. Noting that $\left\langle\nu_{1}^{\prime} \nu_{2}^{\prime}\right\rangle \approx \psi /\left(1-\gamma^{2}\right)$, we immediately see that the two-point correlation function for regions above threshold becomes $\xi_{\mathrm{pk}}=\left(\nu_{t}^{\prime}\right)^{2} \psi /\left(1-\gamma^{2}\right)$, giving a bias $b_{\mathrm{L}}=\nu_{t}^{\prime} /\left(\sigma \sqrt{1-\gamma^{2}}\right)$, i.e. Eqn. (8) .

The bias of halos found in our simulation matches Eqn. (8) quite well. For a power spectrum index $n=-2$, as we have used, note that $\sigma_{s}=\sigma / \sqrt{6}$ and $\gamma=-1 / \sqrt{6}$, for a top-hat window function. In figure 2 we plot the halo bias as a function of peak curvature, along with our prediction from Eqn. (8). There is evidently a strong dependence of bias upon peak curvature. The $\sim 40 \%$ range in $b$ corresponds to a factor of 2 variation in the correlation function for halos of this mass.

One subtlety which we have glossed over in computing the statistics of high-mass peaks is the issue of the ap- 


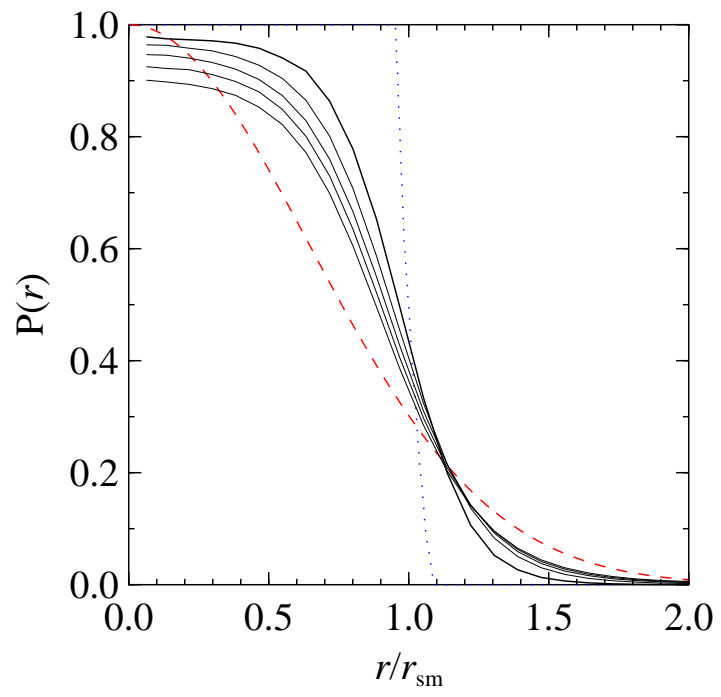

FIG. 3.- Probability for a particle to be linked into a high mass FOF group as a function of initial Lagrangian radius. The thick solid black curve shows the stacked Lagrangian volumes for halos in the mass range 200-300 particles at $a=0.1089$, corresponding to roughly $\sim 3000 M_{\star}$. Note that mass in subhalos is excluded from this average. The blue dotted curve shows the expected average profile for a tophat window, while the red dashed curve corresponds to a Gaussian of this mass range. The thin solid black curves show the average Lagrangian profiles for halos of mass between 200-300 particles at later outputs, $a=0.1896,0.3300,0.5547$, and 1.0000 , corresponding to $M / M_{\star} \simeq 100,4,0.2$ and 0.005 respectively.

propriate smoothing to use. We have assumed a top-hat smoothing, however there is no compelling theoretical reason to favor a top-hat kernel versus other possibilities, such as a Gaussian (although the sharp $k$-space filter implicit in the Press-Schechter model appears unphysical). As a sanity check on our top-hat smoothing, we have computed the average Lagrangian volumes corresponding to high peaks, by stacking roughly 1000 halos of mass $\sim 3000 M_{\star}$. The results are shown in figure 3 The average Lagrangian volume occupied by FOF halos is reasonably compact, with the probability for halo inclusion steeply varying from near 1 to near 0 over a range $0.8<r_{\mathrm{TH}}<1.2$, where $r_{\mathrm{TH}}$ is the top-hat smoothing radius for mass $M$. In comparison, a Gaussian smoothing window is clearly more diffuse. We conclude that our use of top-hat smoothing is reasonable, though we caution that these results may depend upon the slope of the power spectrum. Finally, we note that there is a nontrivial amount of mass inside $r<r_{\mathrm{TH}}$ that does not end up in the halo; we will return to this point below in section 5

\subsection{Halo observables}

We have shown that there is a strong dependence of halo clustering upon peak curvature. How does this relate to the assembly bias observed in previous simulations?

In the high mass regime, there is a direct relationship between peak curvature and halo assembly history. Recall that in the spherical collapse model, regions complete their collapse once the average linearly evolved overdensity $\delta$ reaches $\delta_{c}$. This implies that we can estimate the assembly history of halos simply by measuring how the smoothed overdensity $\langle\delta\rangle$ varies with smoothing scale. We would expect the accretion rate

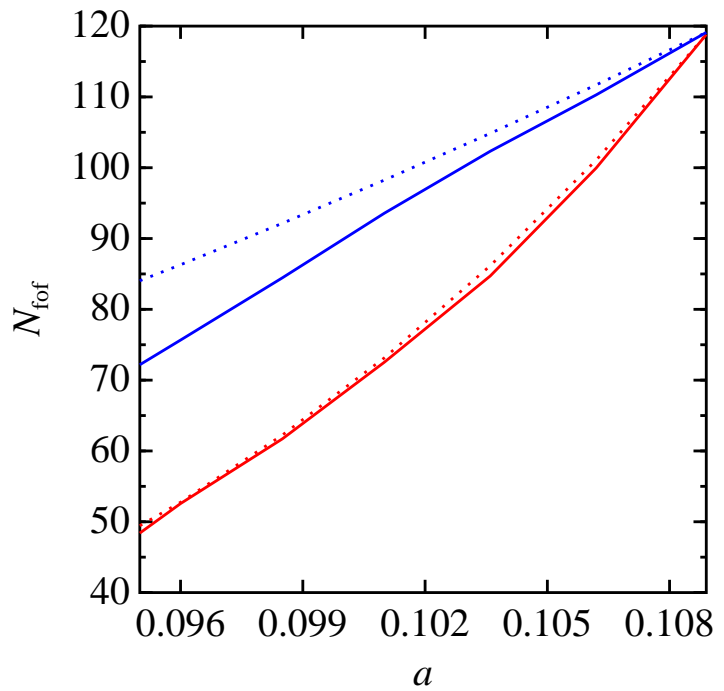

Fig. 4.- Mass accretion histories as a function of peak curvature. The upper solid (blue) curve shows the average accretion history for the halos comprising the leftmost bin in Fig. 2 while the lower solid (red) curve shows the average history for the rightmost bin. The dotted curves depict the behavior $M \propto a^{\alpha}$ for $\alpha=-1 /(d \log \delta / d \log M)$ expected from the spherical collapse model.

and the curvature to be related by $d(\log M) / d(\log a)=$ $-[d(\log \delta) / d(\log M)]^{-1}$. The average mass accretion histories for high mass halos appears consistent with this expectation. In figure 4 we plot the stacked mass accretion history profiles for halos of 120 particles at $a=0.1089$, splitting the population based on peak curvature. The average accretion rates are in good agreement with our expectations. This is true only in the average, however : individual accretion histories are noisy because much of the accretion onto halos is not smooth, but rather consists of discrete accretion events. This may explain why different age indicators for halos give different levels of assembly bias (Wetzel et al. 2007). The noisiness of commonly used age indicators may be large enough to mask the strong assembly bias clearly present in high mass halos.

Another halo parameter seen to correlate with bias is concentration (Wechsler et al. 2006). While we cannot write down an expression directly relating peak curvature and halo concentration, nevertheless we would expect curvature and concentration to be correlated. Previous work has shown that assembly history and concentration are correlated (Wechsler et al. 2002; Zhao et al. 2003a, b) , so the relation between peak curvature and accretion rate discussed above suggests that concentration and peak curvature may be correlated. Physically, we would expect that highly curved peaks should produce more concentrated halos. To test this, we plot in figure 5 the stacked radial profiles of halos with 40005000 particles at $a=0.2501$, as a function of Lagrangian peak curvature. There is a correlation between initial peak curvature and final halo concentration apparent in the stacked profiles, although it is quite apparent that peak curvature is not equivalent to concentration. While the inner profiles (at $r<r_{s}$ ) of curvature-selected halos match well with profiles of concentration-selected halos, the outer profiles (at $r>r_{s}$ ) begin to diverge.

Within the statistical errors of our finite sample of rare 


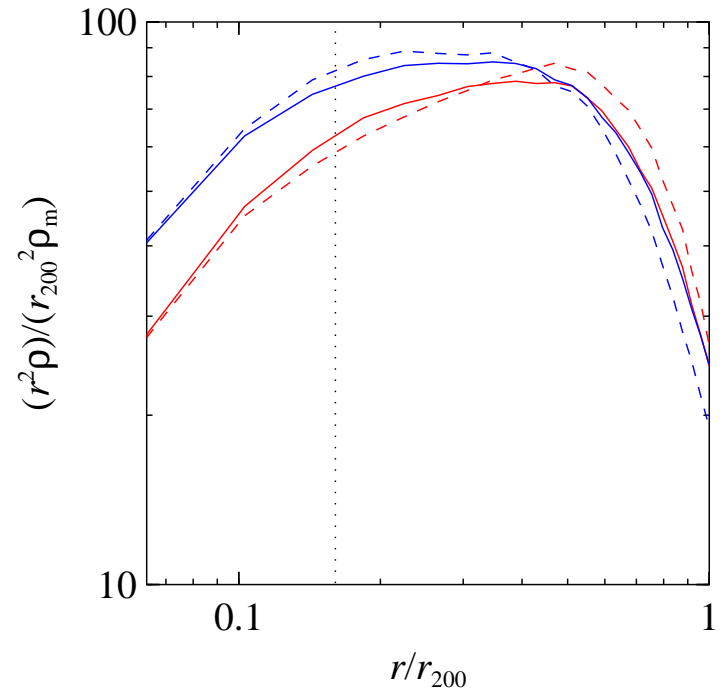

FiG. 5.- Radial profiles for halos as a function of peak curvature. The various curves show the stacked radial profiles for subsets of halos of 4000-5000 particles at $a=0.2501$, roughly $M \sim 400 M_{\star}$. The solid curves correspond to the upper and lower quartiles of $d \log \delta / d \log M$, while the dashed curves correspond to the upper and lower quartiles of fitted concentration $c_{200}$. For comparison, the vertical dotted black line shows $r=2.8 \varepsilon_{P}$, the softening length.

halos, we have found no residual dependence of bias on concentration, once we account for the peak curvature dependence described above. Our simulation had relatively low force resolution, and so our fitted concentrations may be sufficiently noisy to mask any underlying residual concentration dependence of bias, but it appears that assembly bias in rare, high mass halos is in agreement with theoretical expectations. The interpretation of this effect is straightforward: halos residing in high density environments have an enhanced mass supply, and therefore grow faster, than halos in low density environments.

\section{LOW MASS HALOS: $M \ll M_{\star}$}

In this section we consider assembly bias for low mass halos with $M \ll M_{\star}$, for which $\sigma(M) \gg \delta_{c}$. The ageand concentration-dependence of bias at low masses is considerably stronger than in the high mass regime, and of the opposite sign: the oldest, highly concentrated halos have a bias more than twice as large as that of the youngest, low-concentration halos of similar mass $M \ll M_{\star}$ (Gao. Springel \& White 2005; Wechsler et al. 2006; Gao \& White 2007).

In agreement with previous workers, we also find this reversed sense of assembly bias. In keeping with our Lagrangian viewpoint, we use mean Lagrangian overdensity $\langle\delta\rangle$ as a proxy for age. In figure 6, we plot average accretion histories for halos of 200 particles at $a=1$, split into quartiles of $\langle\delta\rangle$. The oldest halos show little growth at late times, accreting only $\sim 20 \%$ of their mass after $a=0.5$. In comparison, the nonlinear mass scale increases by a factor $\approx 64$ over this same time.

Because the oldest halos do not grow appreciably in mass, we can think of their dynamics as those of test particles following large-scale bulk flows. As discussed in section 2 we therefore expect the bias of this population to relax over time to $b \rightarrow 1$. This is indeed the behavior observed for our oldest halos, as shown in figure 7.

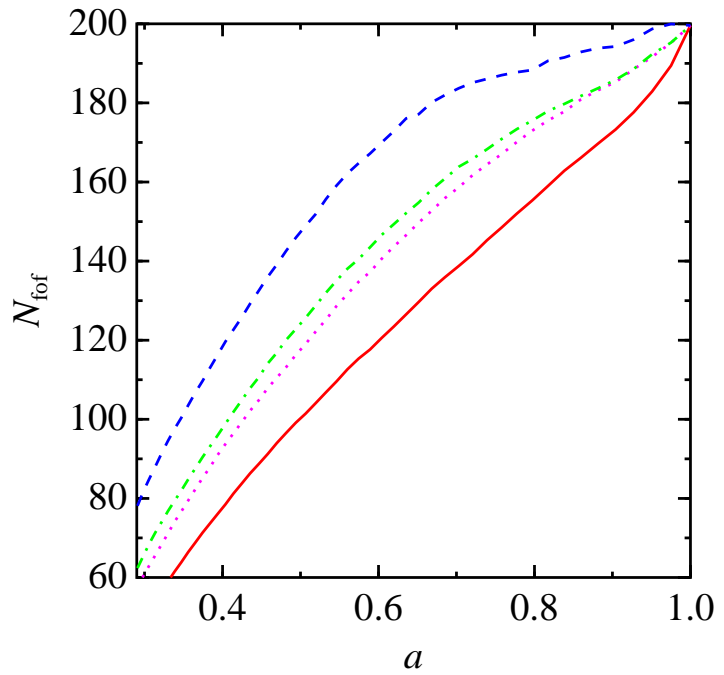

FIG. 6.- Mass accretion histories for halos of 190-210 particles at $a=1$, corresponding to $M=0.0045 M_{\star}$. The halos are split into quartiles of $\langle\delta\rangle$, which correlates well with mass accretion history.

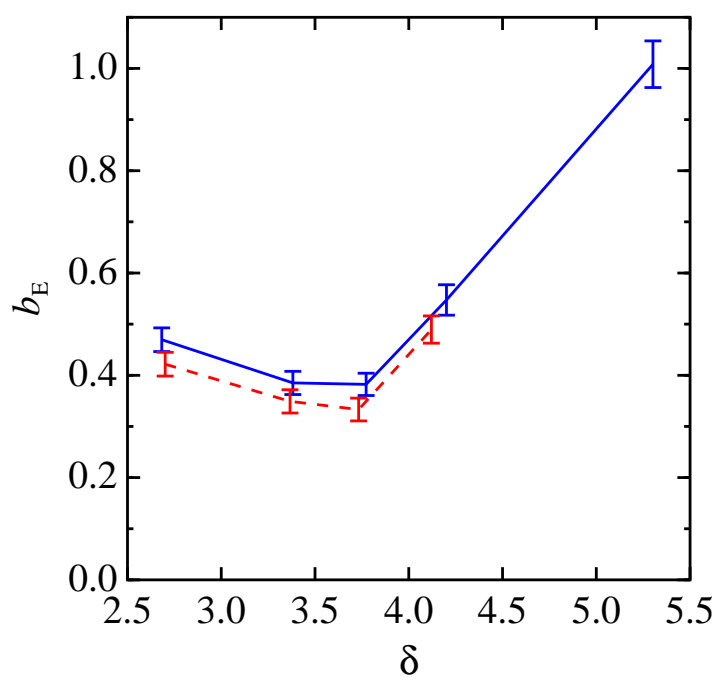

FIG. 7. - Eulerian bias for the same halos of $\sim 200$ particles shown in Figure 6] The oldest halos, at high $\langle\delta\rangle$, are nearly unbiased while the rest of the population is strongly antibiased. Removing all halos which previously were subhalos, and all halos bound to more massive halos, removes the old unbiased subpopulation (red dashed curve).

which plots the (Eulerian) bias of the same halos shown in figure 6. The oldest $\sim 20 \%$ of halos are nearly unbiased, while the remainder are strongly anti-biased, to a degree consistent with predictions of even the simplest Press-Schechter model, $b \rightarrow 1-\delta_{c}^{-1} \approx 0.4$ (Cole \& Kaiser 1989).

The magnitude of assembly bias present at low masses is therefore not surprising. Most low-mass halos are antibiased at expected levels, while a subpopulation becomes unbiased. The theoretical challenge is to explain the number of unbiased, low-mass halos.

One hint towards the origin of the non-accreting, unbiased population comes from the observation that many, if not most of these halos are associated with nearby larger halos (e.g. Wang et al. 2007). In figure 7] we again plot the bias of $\sim 200$ particle halos, but excluding those which in earlier outputs were subhalos. This removes 


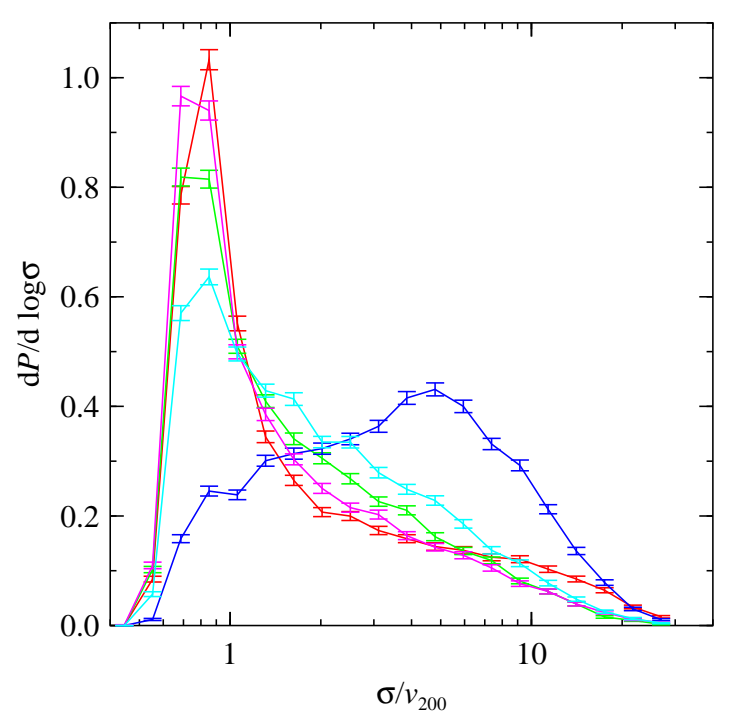

FIG. 8.- Probability distribution for ambient 3-D velocity dispersion $\sigma$ within a radius $5 r_{200}$ of halos with 200-300 particles at $a=1$, roughly $0.005 M_{\star}$. Halos are split into 5 bins of $\langle\delta\rangle$, with red, magenta, green, cyan, and blue from lowest to highest respectively. The oldest halos tend to live in hot environments, whereas the material in the vicinity of most low-mass halos is sufficiently cold to permit accretion.

$\sim 20-25 \%$ of the total population. Because the time sampling of our simulation outputs do not finely cover the dynamical times of all halos, this cut could miss subhalos with orbital pericenters inside of larger halos, but apocenters well outside. Ludlow et al. (2008) have recently argued that a significant fraction of subhalos have orbital apocenters extending out to many times the virial radius of their host halo. To account for this, we also conservatively exclude all halos which are within $3 r_{\text {vir }}$ and gravitationally bound to larger halos. This will exclude the population discussed by Ludlow et al. (2008), as well as any halos entering the infall region of massive objects. This cut removes a further $\sim 15 \%$ of low mass halos, leaving $\gtrsim 60 \%$ of the original low-mass population. The effect of these cuts is effectively to exclude the old subpopulation, even though the cuts do not explicitly refer to halo age or accretion history. It therefore does seem likely that, as suggested by previous authors, the old subpopulation of low-mass halos corresponds to early-formers whose growth was stunted by environmental effects (Wang et al. 2007).

One plausible environmental effect that would account for the arrested development of the oldest low-mass halos is simply the enhanced velocity dispersion in the vicinity of massive halos. In environments where the ambient velocity dispersion greatly exceeds the escape velocity of low-mass halos, those halos will be unable to accrete significant material. We indeed find that the oldest halos preferentially reside within hot environments, as shown in Fig. 8

Given that the oldest low-mass halos tend to exist in proximity to larger halos, we would expect them to originate (in Lagrangian space) from the vicinities of high mass halos as well, c.f. Fig. 3. As discussed in the previous section, much of the material within the Lagrangian volumes of high mass halos ends up within the final collapsed halo, so how do we understand the survival of the old low-mass halos in the presence of their rapidly

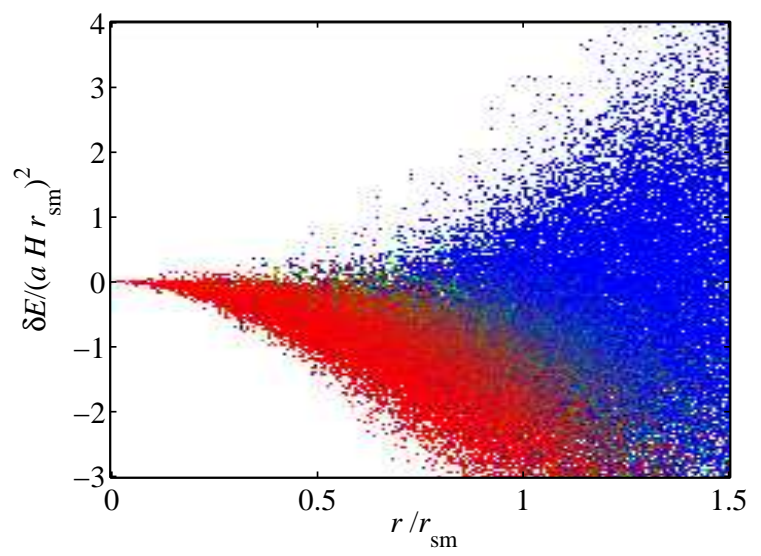

FIG. 9.- Binding energy versus initial radius for peaks that are accreted (red), that become subhalos (green), or that escape (blue).

accreting neighbors? One possible explanation noted by Ludlow et al. (2008) is that these halos are ejected out of larger halos by 3-body interactions. Because our goal is to understand the clustering of halos in terms of their Lagrangian properties of known (Gaussian) statistics, and since complicated multi-body interactions are clearly difficult to model using only Lagrangian quantities, we have not attempted to address this mechanism using our simulations. Rather, we have tried to find Lagrangian properties of halos which correlate with their survival around larger structures.

One Lagrangian quantity which correlates with survival is plotted in Figure 9. We have taken halos of mass 40,000-60,000 particles at $a=1$, nearly $M \simeq M_{\star}$, and identified smaller-scale peaks within the spherical Lagrangian volumes centered on the larger halos. As shown in the figure, the relative binding energy of the subpeaks roughly correlates with their escape likelihood. We have defined the binding energy $\delta E$ in the following manner. The gravitational potential near a peak may be written

$$
\phi(\boldsymbol{x}) \approx \phi_{0}+\boldsymbol{x} \cdot \nabla \phi+\frac{1}{2} \boldsymbol{x} \cdot \nabla \nabla \phi \cdot \boldsymbol{x}+\ldots
$$

The first two terms do not affect the collapse of the peak, and so we define the perturbed potential energy as the quadratic term,

$$
\delta \phi(\boldsymbol{x})=\frac{1}{2} \boldsymbol{x} \cdot \nabla \nabla \phi \cdot \boldsymbol{x}=\frac{3 \Omega H_{0}^{2}}{4 a} \boldsymbol{x} \cdot \mathbf{S} \cdot \boldsymbol{x}
$$

where we have used the Poisson equation to relate the strain tensor $\mathbf{S}=\nabla \nabla\left(\nabla^{-2} \delta\right)$ to the potential's curvature tensor. We can similarly write the kinetic energy near the peak as

$$
\begin{aligned}
T(\boldsymbol{x}) & =\frac{1}{2}|\boldsymbol{v}|^{2}=\frac{1}{2}\left|a H(\boldsymbol{x}+\boldsymbol{d})+\boldsymbol{v}_{p}\right|^{2} \\
& \approx \frac{1}{2}(a H|\boldsymbol{x}|)^{2}+2(a H)^{2} \boldsymbol{x} \cdot \boldsymbol{d}+\ldots
\end{aligned}
$$

where we have used the Zeldovich approximation for $\Omega_{m}=1$ to relate the peculiar velocity and comoving displacement, $\boldsymbol{v}_{p}=a H \boldsymbol{d}$. The unperturbed piece again does not affect the peak dynamics, and so we write the 
perturbed kinetic energy as

$$
\delta T=2(a H)^{2} \boldsymbol{x} \cdot \boldsymbol{d},
$$

and the total perturbed energy becomes $\delta E=\delta \phi+\delta T$. In computing $\delta \phi$, we smooth the strain tensor $\mathbf{S}$ over the large halo, whereas in $\delta T$ we use the difference in the mean Lagrangian peculiar velocities, $\Delta \boldsymbol{v}_{\mathrm{L}}=\boldsymbol{v}_{\mathrm{L} 2}-\boldsymbol{v}_{\mathrm{L} 1}$.

As can be seen from Figure 9, the initial Lagrangian binding energy does not solely determine the survival of subpeaks; the escape threshold is radially dependent as well. Without any physical justification, we find that an approximate threshold

$$
E^{\prime} \equiv \delta E+(a H)^{2}\left[2 r^{2}-r_{\mathrm{sm}}^{2}\right]=0
$$

roughly separates accreted subpeaks from escaping subpeaks.

Our assumption, therefore, is that subpeaks inside of larger peaks can escape accretion onto their hosts if the relative energy is large enough, $E^{\prime}>0$. Due to environmental effects, however, they are unable to grow, and thereby produce the population of old, unbiased lowmass halos. In the next section, we test how well this simple picture can reproduce our numerical bias results.

\section{A TOY MODEL FOR ASSEMBLY BIAS}

In the previous two sections, we have discussed assembly bias in two asymptotic regimes, $\sigma \ll 1$ and $\sigma \gg \delta_{c}$. We found that two distinct effects generated assembly bias in these two regimes. At intermediate masses, $\sigma \sim 1$, both effects can be significant, and the overall bias of a sample of halos will involve a competition between these two effects. The transition between the high-mass behavior and the low mass behavior will depend in detail upon the precise selection criteria used to define the sample of halos. For example, we plot in Figure 10 the halo bias for halos selected on peak height $\langle\delta\rangle$ and on peak curvature $d \log \langle\delta\rangle / d \log M$. Recall that these peak properties both affect halo properties like age and concentration. At high masses, the scatter in peak height among peaks above threshold is small, and so the scatter in peak curvature dominates the assembly bias. At low mass, the peak height is the dominant factor determining halo age or concentration, causing a reversal in the sense of assembly bias. To illustrate, we plot in the right panel of Fig. 10] the bias for halos selected on $\langle\delta\rangle / \delta_{c}-d \log \langle\delta\rangle / d \log M$. At high masses, the curvature term dominates, while at low masses, the first term dominates. Halo samples selected on formation time or concentration show similar behavior. At intermediate masses, both of these effects are significant and compete with each other, and the magnitude and sign of assembly bias will depend in detail upon the precise age indicator that is employed. For example, an age indicator that correlates more strongly with peak height rather than peak curvature will show a crossover at higher masses than the example shown in Fig. 10k. This may explain why different measures of halo age give different amounts of assembly bias when applied to the same samples of halos (Li et al. 2008).

Using our results on the assembly bias of low- and high-mass halos, we have constructed a simple toy model for halo biasing. We generate a Gaussian random density field, smooth the field on multiple scales, and starting from the largest smoothing scales, search for density peaks exceeding the ellipsoidal collapse threshold
(Bond \& Myers 1996, hereafter BM96). Isolated peaks above threshold are labeled as collapsed halos, whereas peaks falling within 1.2 smoothing radii (c.f. Fig. 9) of larger scale collapsed regions are only labeled as collapsed if their binding energies relative to their larger hosts exceeds the threshold found empirically from our N-body halos, i.e. Eqn. (13).

A key component of this calculation is the collapse threshold. A comparison of the simple BM96 model with our N-body results shows that the model works well at predicting collapse thresholds for reasonably high peaks, $\sigma \lesssim 1$, however at low peaks $\sigma \gg 1$, the model begins to break down. Very roughly, the ellipsoidal collapse threshold is larger than the spherical collapse threshold by a ratio $\delta_{\mathrm{ec}} / \delta_{\mathrm{sc}} \sim\left(1-\frac{1}{2} \delta_{\mathrm{sc}} e_{v}\right)^{-1}$, where the ellipticity $e_{v}$ of the strain tensor is given by the difference between its largest and smallest eigenvalues, $e_{v}=\left(S_{33}-S_{11}\right) / 2 \delta$. The ellipticity and prolaticity are random variables whose probability distribution is related to peak height (Bardeen et al. 1986, BM96), but very roughly the mean ellipticity scales as $\left\langle e_{v}\right\rangle \sim 1 /(2 \nu)$. For $\nu<1$, the BM96 collapse threshold quickly grows, and for sufficiently large $e_{v}$ and $-p_{v}$ the model predicts that collapse never occurs. However we find many low mass halos with average peak heights $\langle\delta\rangle$ falling far below the collapse thresholds expected given their average Lagrangian strain tensors $\langle\mathbf{S}\rangle$. This is not surprising. As noted explicitly by BM96, their homogeneous ellipsoid model assumes that the evolution of the external tidal field acting upon the collapsing peak may be determined from the strain tensor $\langle\mathbf{S}\rangle$ averaged over the local volume of the peak. While it is certainly true that the external tidal field and local strain tensor are closely related at early times, in the nonlinear regime the one-to-one correspondence between them breaks down. On scales where $\sigma \gg 1$, the external tidal field evolves nonlinearly in a manner that cannot be predicted from purely local measurements over the peak volume. Therefore, we would not expect the simple BM96 model to apply in this regime, as those authors note.

Nevertheless, we require some choice of collapse threshold for our toy model. At low masses, the lowest $\langle\delta\rangle$ 's are roughly $\sim 2.5$ (see Fig. 7), so we arbitrarily set a threshold $\delta_{c}=\min \left(\delta_{\mathrm{BM} 96}, 2.5\right)$. In addition, we also require that subpeaks collapsing inside of larger peaks must complete their collapse sufficiently before the larger peak collapses. We can estimate the collapse redshift $z_{c}$ by $1+z_{c}=\langle\delta\rangle / \delta_{c}$. Writing $z_{s}$ and $z_{b}$ as the collapse redshifts for the sub-peak and large peak, respectively, we require $\left(1+z_{s}\right) \geq 2\left(1+z_{b}\right)$ for a subpeak to be labeled as a collapsed halo.

Figure 11] shows results of this simple calculation. In order to compare with Fig. 10, we plot bias as a function of the same peak parameters. At high masses, $\sigma \ll 1$, the bias matches Eqn. 8, as it must. At low masses $(\sigma \gg$ 1 ) the bias of isolated peaks asymptotes to $b_{\mathrm{E}} \approx 0.4$, while the subpeaks become unbiased, $b_{\mathrm{E}} \rightarrow 1$. These features appear fairly generic. Behavior in the transition region, $\sigma \sim 1-2$, depends upon details of the model. In particular, the bias of the old halos in the intermediate mass regime depends upon the fraction of halos that are subpeaks, and varying parameters in the model changes this fraction.

Overall, however, we find reasonable agreement be- 

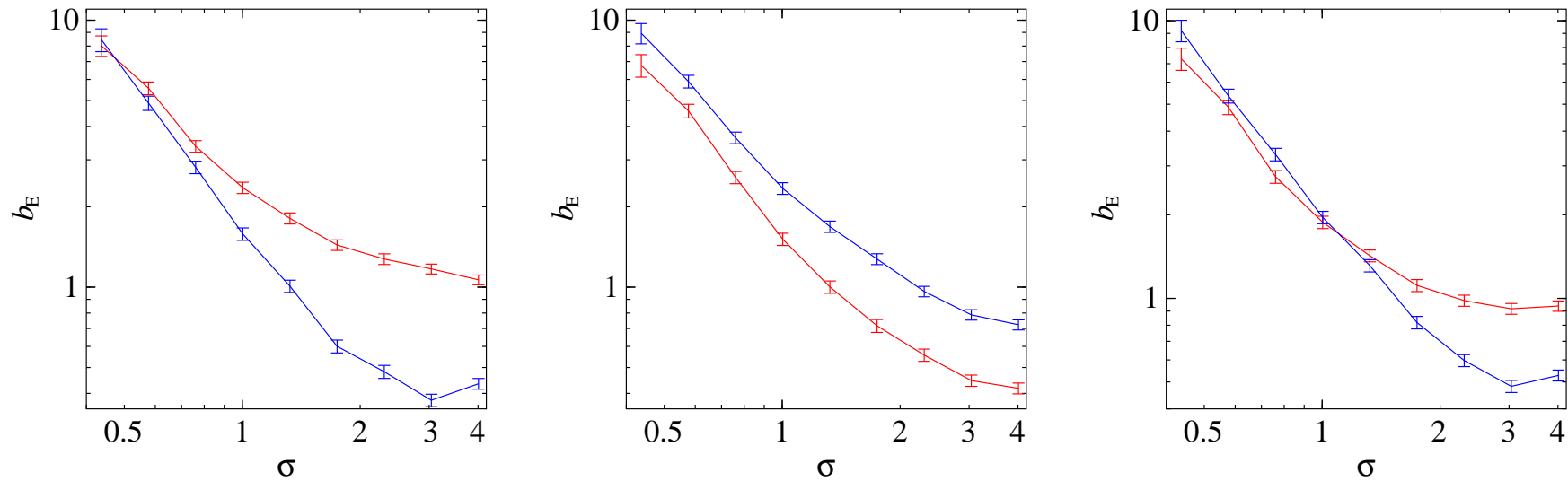

Fig. 10. - Bias as a function of peak parameters. Bias is plotted for halos in the range 200-300 particles at various redshifts. In the left panel, the red and blue curves show bias for the upper and lower $20 \%$ of peak height $\langle\delta\rangle / \delta_{c}$, where $\delta_{c}$ is the ellipsoidal collapse threshold calculated from each halo's local strain tensor. In the middle panel, we split halos on peak curvature, $d \log \langle\delta\rangle / d \log M$. In the right panel, we split halos on an average of these two quantities, $\langle\delta\rangle / \delta_{c}-d \log \langle\delta\rangle / d \log M$. At high masses, the scatter in curvature dominates, while at low masses, the scatter in peak height dominates, leading to a transition in assembly bias at intermediate masses. Formation time or concentration shows similar behavior.
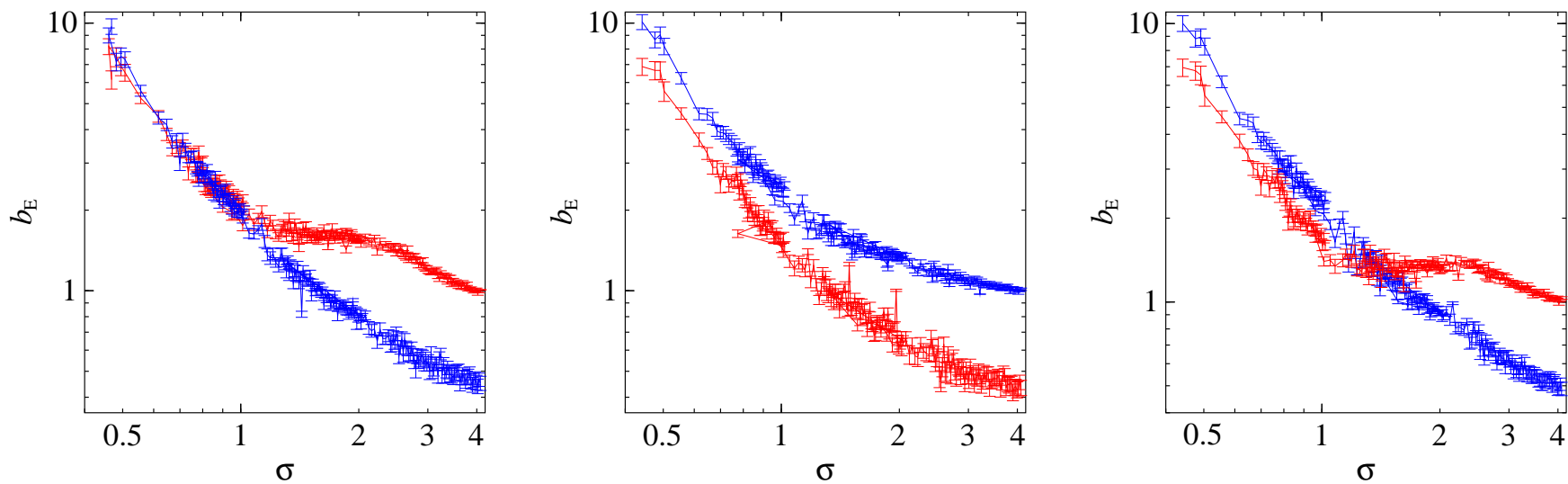

Fig. 11. - Similar to Fig. 10 but for peaks found in our toy model calculation rather than N-body halos.

tween the toy model and our N-body simulation in the regimes where assembly bias is significant, for $M \gg M_{\star}$ and $M \ll M_{\star}$. The point of this exercise is not to claim that we have solved the halo formation problem, but merely to illustrate that the assembly bias in these regimes is generic. The assembly bias at high masses is an unavoidable consequence of Gaussian statistics, and therefore any halo formation model which relates initial density peaks to halos will also find similar levels of assembly bias, depending somewhat on the peak definition. The behavior at low masses may appear more model dependent, however we do not believe our results are specific to our particular mechanism for production of non-accreting halos. Rather, we expect that any mechanism which produces roughly the correct fraction of nongrowing, low-mass halos will also match the assembly bias found in N-body simulations.

\section{DISCUSSION \& CONCLUSIONS}

We have investigated halo assembly bias in hierarchical structure formation. We find that assembly bias is significant both at high masses $\left(M \gg M_{\star}\right)$ and low masses $\left(M \ll M_{\star}\right)$. Two different competing effects generate assembly bias in these two regimes. At high masses, peaks of low curvature are more strongly clustered than peaks of large curvature, in a manner entirely consistent with the statistics of peaks of random Gaussian fields. We provide a simple expression for the bias in the high-mass regime which accounts for assembly bias and reduces to the Press-Schechter bias when marginalized over all parameters excluding mass (note that fitting formulae for bias calibrated at lower mass, such as those proposed by Sheth \& Tormen (1999) or Seliak \& Warren (2004), fail in the high mass regime (Cohn \& White 2007)). At low masses, assembly bias appears largely driven by the formation of a non-accreting sub-population of low-mass halos in the vicinity of larger halos. Because the nonaccreting halos become unbiased, they are more strongly clustered than accreting halos of similar mass, which are anti-biased. We found that a simple toy model is able to reproduce our numerical bias results in the asymptotically large and small mass regimes. At intermediate masses $\left(M \sim M_{\star}\right)$, both of the above effects become important, and the assembly bias for a sample of halos will involve a weighted average of both effects.

The application of these results to observed objects like galaxies or quasars is not straightforward, because it will depend upon the halo occupation distribution (HOD) of those objects. The very simplest HOD models assume that the galaxy content depends only upon halo mass, and not upon assembly history, whereas semianalytic models used in conjunction with N-body simu- 
lations tend to find a strong dependence of galaxy properties on assembly history at fixed mass (Springel et al. 2005; Croton et al. 2007, e.g.). If the former assumption is valid, then assembly bias will not be important for galaxy clustering. Perhaps surprisingly, Tinker et al. (2007) have recently found that properties of observed SDSS galaxies do not appear to correlate significantly with local environment, once the dependence on host halo mass has been accounted for, suggesting that assembly bias may indeed be unimportant for modeling galaxy clustering.

Besides the clustering of halos, our results also have implications for our understanding of halo assembly. In particular, we have found that massive halos are very well described by spherical collapse. The average precollapse Lagrangian volumes occupied by the most massive halos are approximately spherical regions of size $r \sim r_{\mathrm{TH}}=(3 M / 4 \pi \rho)^{1 / 3}$. However, $r_{\mathrm{TH}}$ is not a sharp boundary in Lagrangian space: there is significant mass outside $r>r_{\mathrm{TH}}$ at early times which is found inside the virial radius at late times, and similarly there is consid- erable mass at $r<r_{\mathrm{TH}}$ which avoids accretion at late times. Indeed, we might speculate that the post-collapse radial distribution of the matter inside and around a dark matter halo is simply related to the pre-collapse profile of local density and potential. Since both of these quantities are described by well-understood Gaussian statistics, this raises the intriguing possibility of understanding the internal structure of virialized objects from first principles. This topic is the subject of further study.

We thank Andrey Kravtsov and Simon White for several helpful discussions. We also thank Joanne Cohn, Andrey Kravtsov and Andrew Wetzel for comments on an earlier version of this paper. All simulations were performed on CITA's Sunnyvale cluster, funded by the Canada Foundation for Innovation and the Ontario Research Fund for Research Infrastructure. N.D. was supported by the Natural Sciences and Engineering Research Council of Canada (NSERC). M.W. was supported in part by NASA.

\section{REFERENCES}

Allgood, B., Flores, R. A., Primack, J. R., Kravtsov, A. V., Wechsler, R. H., Faltenbacher, A., \& Bullock, J. S. 2006, MNRAS, 367, 1781

Angulo R.E., Baugh C.M., Lacey C.G., 2008, preprint arXiv:0712.2280

Avila-Reese, V., Colín, P., Gottlöber, S., Firmani, C., \& Maulbetsch, C. 2005, ApJ, 634, 51

Bett P., Eke V., Frenk C.S., Jenkins A., Helly J., Navarro J., 2007, MNRAS, 376, 215

Bardeen J.M., Bond J.R., Kaiser N., Szalay A.S., 1986, ApJ, 304, 15

Bond J.R., Cole S., Efstathiou G., \& Kaiser N. 1991, ApJ, 379, 440

Bond, J. R., Kofman, L., \& Pogosyan, D. 1996, Nature, 380, 603

Bond J.R., Myers S.T., 1996, ApJS, 103, 1

Cohn, J. D., \& White, M. 2007, arXiv:0706.0208

Cole, S. \& Kaiser, N. 1989, MNRAS, 237, 1127

Conroy, C., Wechsler, R.H., \& Kravstov, A.V. 2006, ApJ, 647, 201

Cooray, A. \& Sheth, R. 2002, Phys. Rep., 372, 1

Croton, D. J., Gao, L., \& White, S. D. M. 2007, MNRAS, 374, 1303

Cuesta, A. J., Prada, F., Klypin, A., \& Moles, M. 2007, arXiv:0710.5520

Davis, M., Efstathiou, G., Frenk, C.S., \& White, S.D.M. 1985, ApJ, 292, 371

Desjacques, V. 2007, arXiv:0707.4670

Efstathiou, G., et al. 1988, MNRAS, 235, 715

Faltenbacher A., Allgood B., Gottloeber S., Yepes G., Hoffman Y., 2005, MNRAS, 362, 1099

Fry, J. N. 1996, ApJ, 461, L65

Gao, L., Springel, V., \& White, S.D.M 2005, MNRAS, 363, L66

Gao, L., \& White, S. D. M. 2007, MNRAS, 377, L5

Gunn, J. E., \& Gott, J. R. I. 1972, ApJ, 176, 1

Harker, G., Cole, S., Helly, J., Frenk, C., \& Jenkins, A. 2006, MNRAS, 367, 1039

Jing Y.-P., Suto Y., Mo H.-J., 2007, ApJ, 657, 664

Kaiser, N. 1984, ApJ, 284, L9

Kauffmann, G. \& Haehnelt, M. 2000, MNRAS, 311, 576

Kravtsov, A. V., Gnedin, O. Y., \& Klypin, A. A. 2004, ApJ, 609, 482

Lemson, G. \& Kauffmann, G. 1999, MNRAS, 302, 111
Li, Y., Mo, H. J., \& Gao, L. 2008, arXiv:0803.2250

Lima, M., \& Hu, W. 2004, Phys. Rev. D, 70, 043504

Ludlow, A. D., Navarro, J. F., Springel, V., Jenkins, A., Frenk, C. S., \& Helmi, A. 2008, arXiv:0801.1127

Mo, H. J., Yang, X., van den Bosch, F. C., \& Katz, N. 2005, MNRAS, 363, 1155

Mo, H.J., \& White, S.D.M. 1996, MNRAS, 282, 347

Navarro, J. F., Frenk, C.S., \& White, S.D.M. 1997, ApJ, 490, 493

Peacock, J. A. 1999, Cosmological Physics, Cambridge University Press

Prada, F., Klypin, A. A., Simonneau, E., Betancort-Rijo, J., Patiri, S., Gottlöber, S., \& Sanchez-Conde, M. A. 2006, ApJ, 645, 1001

Press, W. \& Schechter, P. 1974, ApJ, 187, 425

Sandvik, H. B., Möller, O., Lee, J., \& White, S. D. M. 2007, MNRAS, 377, 234

Seljak, U., \& Warren, M. S. 2004, MNRAS, 355, 129

Sheth, R.K. \& Tormen, G. 1999, MNRAS, 308, 119

Sheth, R.K. \& Tormen, G. 2004, MNRAS, 350, 1385

Shirokov, A. \& Bertschinger, E. 2005, astro-ph/0505087

Springel V., White S.D.M., Tormen G., Kauttmann G., 2001, MNRAS, 328, 726 .

Springel V., et al., 2005, Nature, 435, 629

Tegmark M., Peebles P.J.E., 1998, ApJ, 500, L79

Tinker, J. L., Conroy, C., Norberg, P., Patiri, S. G., Weinberg, D. H., \& Warren, M. S. 2007, ArXiv e-prints, 707, arXiv:0707.3445

Wang, H. Y., Mo, H. J., \& Jing, Y. P. 2007, MNRAS, 375, 633

Wechsler, R. H., Bullock, J. S., Primack, J. R., Kravtsov, A. V., \& Dekel, A. 2002, ApJ, 568, 52

Wechsler, R.H., Zentner, A.R., Bullock, J.S., Kravtsov, A.V., Allgood B., 2006, ApJ, 652, 71

Wetzel A.R., Cohn J.D., White M., Holz D.E., Warren M.S., 2007, ApJ, 656, 139

Wu, H.-Y., Rozo, E., \& Wechsler, R. H. 2008, arXiv:0803.1491

Zhao, D. H., Jing, Y. P., Mo, H. J., Borner, G. 2003, ApJ, 597, L9

Zhao, D. H., Mo, H. J., Jing, Y. P., Borner, G. 2003, MNRAS, 339,12 